\title{
De novo biosynthesis of $C$-arabinosylated flavones by utilization of indica rice C-glycosyltransferases
}

\author{
Zhuo Chen ${ }^{1,2 \dagger}$, Yuwei Sun ${ }^{1 \dagger}$, Guangyi Wang ${ }^{1,2}$, Ying Zhang ${ }^{1,2}$, Qian Zhang ${ }^{1,2}$, Yulian Zhang ${ }^{1,2}$, Jianhua Li ${ }^{1}$ and \\ Yong Wang ${ }^{1 *}$ (1)
}

\begin{abstract}
Flavone C-arabinosides/xylosides are plant-originated glycoconjugates with various bioactivities. However, the potential utility of these molecules is hindered by their low abundance in nature. Engineering biosynthesis pathway in heterologous bacterial chassis provides a sustainable source of these $\mathrm{C}$-glycosides. We previously reported bifunctional C-glucosyl/C-arabinosyltransferases in Oryza sativa japonica and O. sativa indica, which influence the C-glycoside spectrum in different rice varieties. In this study, we proved the C-arabinosyl-transferring activity of rice C-glycosyltransferases (CGTs) on the mono-C-glucoside substrate nothofagin, followed by taking advantage of specific CGTs and introducing heterologous UDP-pentose supply, to realize the production of eight different C-arabinosides/xylosides in recombinant E. coli. Fed-batch fermentation and precursor supplement maximized the titer of rice-originated C-arabinosides to 20-110 mg/L in an E. coli chassis. The optimized final titer of schaftoside and apigenin di-C-arabinoside reached 19.87 and $113.16 \mathrm{mg} / \mathrm{L}$, respectively. We demonstrate here the success of de novo bio-production of C-arabinosylated and C-xylosylated flavones by heterologous pathway reconstitution. These results lay a foundation for further optimal manufacture of complex flavonoid compounds in microbial cell factories.
\end{abstract}

Keywords: De novo biosynthesis, C-Arabinoside flavone, C-Glycosyltransferase, Rice

\section{Introduction}

Flavone $C$-arabinosides are one of the less common classes of flavonoid glycosides occurring in nature. Despite of their rarity, many flavones bearing $C$-arabinosyls have been reported to show intriguing physiological activities. For example, schaftoside (apigenin 6-C-glucosyl-8-C-arabinoside, $\mathrm{Sch}$ ) is present as a major component in the Chinese herb Desmodium styracifolium, possessing diverse bioactivities including antioxidant, anti-inflammatory (De Melo et al. 2005), antimelanogenic (Kim et al. 2018b) activities and inhibiting the formation

\footnotetext{
*Correspondence: yongwang@cemps.ac.cn

${ }^{\dagger}$ Zhuo Chen and Yuwei Sun contributed equally to this work

${ }^{1}$ CAS-Key Laboratory of Synthetic Biology, CAS Center for Excellence in Molecular Plant Sciences, Institute of Plant Physiology and Ecology, Chinese Academy of Sciences, Shanghai 200032, China

Full list of author information is available at the end of the article
}

of gallstones and kidney stones (Liu et al. 2017). Besides, schaftoside was also reported as a feeding inhibitor and resistance factor to brown planthopper (Stevenson et al. 1996). As an isomer of schaftoside, isoschaftoside (Isosch) was also found to be an allelochemical against the development of Striga (Hooper et al. 2010). Carlinoside (luteolin 6-C-glucosyl-8-C-arabinoside) from Cajanus plants shows antihepatitic and bilirubin solubilization activity (Das et al. 2018). Recently, both schaftoside and carlinoside were identified as active ingredients against COVID-19. In silico analysis regarded schaftoside as one of the top 10 among 318 phytochemicals that had significantly lower binding energy to Mpro (the main protease of SARSCoV-2) and ACE2 (angiotensinconverting enzyme 2) as compared to the reference molecule PRD_002214 (Joshi et al. 2020). Molecular docking indicated carlinoside was the top candidate against Mpro 
(Ettayapuram Ramaprasad et al. 2020; Joshi et al. 2020). Therefore, flavone $C$-arabinosides are expected to be a powerful weapon for potential treatment of SARSCoV-2.

Largely lagging behind the discovery and bioactivity assay of $C$-arabinosylated flavones, the in planta biosynthesis of $C$-arabinosides was occasionally studied (Putkaradze et al. 2021). At present, only a few $C$-glycosyltransferases (CGTs) that accommodating uridine-5'diphosphate (UDP)-arabinose have been reported (Chen et al. 2018; Feng et al. 2021; He et al. 2019; Sun et al. 2020; Wang et al. 2020; Zhang et al. 2020). In our previous work, a group of gramineae CGTs was identified as glycosyltransferases utilizing UDP-glucose (UDP-Glc) and UDP-arabinose (UDP-Ara) for the $C$-glycosylation of phloretin and 2-hydroxynaringin (2-OHNar) (Sun et al. 2020). It is likely that the grass family plants have evolved two branches of CGTs, in which one group is more specialized for $\mathrm{C}$-glucosylation (designated as clade $\mathrm{A}$ ) and another is more relaxed to accept both UDP-Glc and UDP-Ara donors (designated as clade B). Correspondingly, the chemical diversity of flavone $C$-glycosides in Gramineae family does reflect the promiscuity of their CGTs, as both $C$-glucosyl and $C$-arabinosyl-carrying metabolites were frequently found in these grasses represented by rice (Besson et al. 1985; Melo et al. 2005; Talhi and Silva 2012). Oryza sativa (rice) is an important gramineae crop closely related to the life of billions of people. The leaves of $O$. sativa subsp. japonica accumulate a high proportion of flavone $C$-pentosylhexosides mainly represented by (iso)schaftoside and (iso)carlinoside (Sun et al. 2020). Such metabolite profiles indicate that CGTs from the rice may be excellent candidates for the production of flavone $C$-glycosides, especially flavone di- $C$-glycosides carrying hexosyl (i.e., glucosyl) and pentosyl (i.e., arabinosyl).

We previously discovered that the chromosome 6 of $O$. sativa subsp. indica (long-grain rice) harbors six tandem duplicated CGT-encoding genes, which is twice as many as those of japonica rice (Sun et al. 2020). Sequence analyses implied an expansion of clade B CGTs including 4 members (OsUGT708A1, OsUGT708A2, OsUGT708A39 and OsUGT708A40) (Additional File 1: Fig. S1). Genetic mechanism underlying the varietal differences of distinct rice genotype has been an attractive topic for long years, nevertheless there is still few studies mentioned the variance of rice $C$-glycoside spectrum and genes linked to such phenotypes. It is reasonable to hypothesize that the additional clade B CGTs in indica rice may play an important role in the formation of specific $C$-arabinosides, resulting in intraspecific difference.

At present, large-scale production of flavone $C$-glycosides, especially the rare flavone $C$-arabinosides is exclusively limited to plant extraction. Complex extraction processes and unsustainable source are great challenges to meet the ever-growing demand. Recently there have been some attempts on the production of flavone $\mathrm{C}$-monoglucosides in heterologous chassis cells (BrazierHicks and Edwards 2013; Vanegas et al. 2018; Ito et al. 2014; Shrestha et al. 2018; Sun et al. 2020). However, as far as we know, there have been no reports of de novo biosynthesis of complex flavone (di)-C-glycosides with arabinosyl or other pentosyl moiety. With the development of synthetic biology, production of flavonoid glycosides by heterologous chassis cells become a promising alternative way to access these bioactive molecules at a much lower cost (Kim et al. 2015; Lim et al. 2015; Liu et al. 2018; Malla et al. 2013; Pandey et al. 2013; Pei et al. 2016; Schmidt et al. 2011; Shrestha et al. 2018; Simkhada et al. 2010). In this study, we proved the $C$-arabinosyltransferring activity of rice CGTs on the mono- $C$-glucoside substrate nothofagin, followed by taking advantage of specific CGTs to realize the production of several di$\mathrm{C}$-glycosides including eight different $\mathrm{C}$-arabinosides/ xylosides. The strategy combining heterologous UDPpentose supply, precursor supplement and fed-batch fermentation maximized the titer of rice-originated $C$-arabinosides to $20-110 \mathrm{mg} / \mathrm{L}$ in an Escherichia coli chassis for the time.

\section{Materials and methods}

\section{Plant materials, chemicals}

Oryza sativa subsp. japonica (cv. Nipponbare) and Oryza sativa subsp. indica (cv. Teqing) were grown in CAS Center for Excellence in Molecular Plant Science. Chemical standards including schaftoside (Sch), isoschaftoside (Isosch), phloretin (Phr), vitexin (Vit), isovitexin (Isovit), naringenin (Nar), $p$-coumaric acid (p-CA), vicenin-1 and vicenin-3 were purchased from Dalian Meilun Biotechnology Co., LTD (China). Nothofagin, apigenin 6,8-C-di-arabinoside (Api-di-C-Ara) and apigenin 6,8-C-di-xyloside (Api-di- $C$-Xyl) were prepared by our laboratory and confirmed by NMR analysis. UDPglucose (UDP-Glc, Realtimes Biotechnology Co., Ltd., Beijing, China) and UDP-arabinose (UDP-Ara, CarboSource, U.S.A) were used as sugar donors in the enzymatic assays.

\section{Metabolic analysis of leaves of two rice cultivars}

The frozen leaves of two rice cultivars were extracted as previously described (Sun et al. 2020). Briefly, the crude examples extracted by $75 \%$ methanol were concentrated, re-suspended in water and extracted three times with n-butanol. The organic layer was combined and evaporated to dryness. All samples were analyzed by high-performance liquid chromatography (HPLC) on an Ultimate 3000 HPLC system (ThermoFisher Scientific) with an 
Ecosil 120-5-C18 column $(\phi 4.6 \times 250 \mathrm{~mm}, 5 \mu \mathrm{m})$. The compounds were separated by water (containing $0.1 \%$ formic acid, solvent A) and acetonitrile containing $0.1 \%$ formic acid, solvent B) at a flow rate of $1.0 \mathrm{~mL} / \mathrm{min}$. The samples were eluted under a linear gradient condition: 0.0-16.0 min, $10 \%-25 \% \mathrm{~B} ; 16.0 \mathrm{~min}-30.0 \mathrm{~min}, 25 \%-$ $100 \% \mathrm{~B} ; 30.0 \mathrm{~min}-35.0 \mathrm{~min}, 100 \% \mathrm{~B}$. Ultra-performance liquid chromatography (UPLC)-high-resolution (HR)mass (MS)/MS were acquired using Q Exactive hybrid quadrupole-Orbitrap mass spectrometer (Thermo Scientific, U.S.A.) equipped with an Acquity UPLC BEH C18 column $(\phi 2.1 \times 50 \mathrm{~mm}, 1.7 \mu \mathrm{M}$, Waters, U.S.A.). A linear gradient was set as follows: $0.0-10.0 \mathrm{~min}, 5 \%-95 \%$ acetonitrile $\left(0.1 \%\right.$ formic acid) in $\mathrm{H}_{2} \mathrm{O} ; 10.0-12.5$ min, keep 95\% acetonitrile; $12.5-15.0$, re-equilibrate to the initial condition. The flow rate was $0.25 \mathrm{~mL} / \mathrm{min}$. The mass acquisition was performed in negative ionization mode with full scan (50-1000).

\section{Expression of $C$-glycosyltransferases}

The genomic DNA (gDNA) of O. sativa indica was extracted by the Plant Genomic DNA Kit (Tiangen, Beijing). OsUGT708A1 and OsUGT708A40 were amplified directly from the gDNA by PCR using PrimeSTAR Max DNA polymerase (Takara, Japan). OsUGT708A2 and OsUGT708A39 were synthesized and codon-optimized by Genscript Co. Ltd. (Nanjing, China). The rice CGTs were inserted into NdeI/NotI-double digested pET28a via plus One step PCR Cloning Kit (NovoRec, Shanghai, China) (Additional file 1: Table S1) and transformed into E. coli BL21(DE3) for recombinant expression. Positive clones were grown overnight in $2 \mathrm{~mL}$ Luria-Bertani (LB) media and inoculated into $100 \mathrm{~mL}$ of fresh $\mathrm{LB}$ medium. When the $\mathrm{OD}_{600}$ reached $0.5-0.7,0.1 \mathrm{mM}$ isopropyl $\beta$-D1-thiogalactopyranoside (IPTG) was used to induce the protein expression at $16{ }^{\circ} \mathrm{C}$ for $20 \mathrm{~h}$. The cells were collected by centrifugation ( $6000 \mathrm{rpm}, 5 \mathrm{~min}$ ) and lysed by using a sonication homogenizer (50 W, five cycles). The crude protein extracts were stored at $-20{ }^{\circ} \mathrm{C}$ for subsequent purification. Ni NTA Magarose Beads (Shanghai Chuzhi Biological Technology, Shanghai, China) was used to purify the $\mathrm{His}_{6}$-tagged protein.

\section{In vitro enzymatic assay of CGTs}

A typical enzymatic assay was performed in a $100 \mu \mathrm{L}$ aliquot of reaction mixture containing buffer A $(100 \mathrm{mM}$ $\mathrm{NaCl}, 20 \mathrm{mM}$ Tris-HCl, pH 8.0), $200 \mu \mathrm{M}$ UDP-arabinose, $100 \mu \mathrm{M}$ nothofagin and $25 \mu \mathrm{g}$ purified enzymes. The reaction mixtures were incubated at $37^{\circ} \mathrm{C}$ for $2 \mathrm{~h}$. One hundred microliter of methanol was added to quench the reaction. The mixtures were centrifuged $(12,000 \mathrm{rpm})$ for $15 \mathrm{~min}$ and subjected to HPLC analyses. Separation was achieved on a C18 column [SilGreen ODS column $(\phi 4.6 \times 250 \mathrm{~mm}, \mathrm{~S}-5 \mu \mathrm{M})$, Greenherbs Co., Ltd., Beijing, China] with a flow rate of $1 \mathrm{~mL} / \mathrm{min}$ at $40{ }^{\circ} \mathrm{C}$. Mobile phases contained acetonitrile $(0.1 \%$ formic acid, solvent A) and $\mathrm{H}_{2} \mathrm{O}(0.1 \%$ formic acid, solvent $\mathrm{B})$ under a linear gradient elution: $0-20 \mathrm{~min}, 5 \%$ to $100 \% \mathrm{~A}$ in $\mathrm{B}, 100 \% \mathrm{~A}$ maintained for $5 \mathrm{~min}$. The absorption was monitored at $\lambda=280 \mathrm{~nm}$ and $340 \mathrm{~nm}$.

\section{Reconstruction of $C$-arabinoside pathway}

OsUGT708A1 was inserted into pCZ86 (pET28a harboring PhUGT708A43, Additional file 1: Table S1) between the NotI site via ClonExpress II One Step Cloning Kit (Vazyme, Nanjing, China), resulting in pCZ191. In order to identify an optimal combination for UDP-arabinose biosynthesis, we cloned SmUxs1, SmUxs2 and SmUxe from Sinorhizobium meliloti 1021. AxyPrep Bacterial Genomic DNA Miniprep Kit (Axygen, USA) was used to extract the gDNA of S. meliloti. SmUxs1, SmUxs2 and SmUxe were amplified by PCR using PrimeSTAR Max DNA polymerase (Takara, Japan) with gene-specific primers (Additional file 1: Table S2). We first inserted SmUxs1 and SmUxs2 into BamHI-digested pCZ191 to give pCZ192-1 and pCZ192-2, respectively. SmUxe was further introduced into the BamHI site of pCZ192-1 and pCZ192-2 via ClonExpress II One Step Cloning Kit to accomplish pCZ193-1 and pCZ193-2. The cassette of SmUxs1-SmUxe was amplified using pCZ192-1 as template and inserted into the NotI site of pCZ165 (pET28a harboring OsUGT708A40, Additional file 1: Table S1) to accomplish pCZ194 which was ready for apigenin $C$-arabinoside production. The whole sequence of pCZ194 excepted SmUxe was amplified by reverse PCR to give pCZ195. For the de novo production of flavone $C$-arabinoside and flavone $C$-xyloside from tyrosine, the assembled glycosylation modules (pCZ193-1/pCZ1932/ pCZ192-1/pCZ194/pCZ195) or empty vector $\mathrm{pET} 28 \mathrm{a}$ were co-transferred with pYH055 (Li et al. 2019) and pCZ201 (Sun et al. 2020) into E. coli BL21(DE3) to give strain sCZ113, sCZ114, sCZ115, sCZ118, sCZ119 and sCZ110 (as a negative control).

\section{Fermentation of $C$-arabinosylated flavones}

For flask-shake fermentation, the seeds were precultured at $37{ }^{\circ} \mathrm{C}$ in Luria broth (LB) medium overnight and then inoculated (1:100) into MOPS minimal medium supplemented with $5 \mathrm{~g} / \mathrm{L}$ glucose. After the $\mathrm{OD}_{600}$ reached to 1.0, IPTG $(0.1 \mathrm{mM})$ and tyrosine $(0.5 \mathrm{~g} / \mathrm{L})$ was added to the cultures. Subsequently, the cultures were incubated at $22{ }^{\circ} \mathrm{C}, 250 \mathrm{rpms}$ and maintain for $96 \mathrm{~h}$.

For bioreactor fermentation, the seeds were precultured at $37^{\circ} \mathrm{C}$ in LB medium overnight and then inoculated (1:100) into $100 \mathrm{~mL} \mathrm{LB}$ medium for $5 \mathrm{~h}$. Then all $100 \mathrm{~mL}$ seeds were inoculated into a 5 -L bioreactor 
(Biostat B plus, BioSartorius Stedim Biotech, Germany) at $37{ }^{\circ} \mathrm{C}$, which contained $1 \mathrm{~L}$ M9 minimal media $(15.7 \mathrm{~g} / \mathrm{L}$ $\mathrm{K}_{2} \mathrm{HPO}_{4} \cdot 3 \mathrm{H}_{2} \mathrm{O}, 4.2 \mathrm{~g} / \mathrm{L} \quad \mathrm{KH}_{2} \mathrm{PO}_{4}, 2 \mathrm{~g} / \mathrm{L} \quad\left(\mathrm{NH}_{4}\right)_{2} \mathrm{SO}_{4}$, $1.8 \mathrm{~g} / \mathrm{L}$ citric acid, $1.2 \mathrm{~g} / \mathrm{L} \mathrm{MgSO}_{4} \cdot 7 \mathrm{H}_{2} \mathrm{O}, 0.5 \mathrm{~g} / \mathrm{L}$ yeast extract) with $20 \mathrm{~g} / \mathrm{L}$ glucose, $5 \mathrm{~mL} / \mathrm{L}$ of trace metal solution and $100 \mu \mathrm{L}$ antifoam 204. The trace metal solution contained $0.5 \mathrm{M} \mathrm{HCl}, 10 \mathrm{~g} \mathrm{FeSO}_{4} \cdot 7 \mathrm{H}_{2} \mathrm{O}, 2 \mathrm{~g} \mathrm{CaCl}_{2}, 2.2 \mathrm{~g}$ $\mathrm{ZnSO}_{4} \cdot 7 \mathrm{H}_{2} \mathrm{O}, 0.5 \mathrm{~g} \mathrm{MnSO}_{4} \cdot 4 \mathrm{H}_{2} \mathrm{O}, 1 \mathrm{~g} \mathrm{CuSO}_{4} \cdot 5 \mathrm{H}_{2} \mathrm{O}, 0.1 \mathrm{~g}$ $\left(\mathrm{NH}_{4}\right)_{6} \mathrm{Mo}_{7} \mathrm{O}_{24} \cdot 4 \mathrm{H}_{2} \mathrm{O}$, and $0.02 \mathrm{~g} \mathrm{Na}_{2} \mathrm{~B}_{4} \mathrm{O}_{7} \cdot 10 \mathrm{H}_{2} \mathrm{O}$ per liter of solution. When the $\mathrm{OD}_{600}$ increased to 10 , IPTG $(0.5 \mathrm{mM})$ and tyrosine $(1 \mathrm{~g} / \mathrm{L})$ was added to the cultures and the $\mathrm{pH}$, temperature and dissolved oxygen (DO) were automatically maintained at $7.0,22{ }^{\circ} \mathrm{C}$ and $30 \%(\mathrm{v} / \mathrm{v})$. The feeding solution contained $500 \mathrm{~g} / \mathrm{L}$ glucose, $10.7 \mathrm{~g} / \mathrm{L}$ $\left(\mathrm{NH}_{4}\right)_{2} \mathrm{SO}_{4}, 12 \mathrm{~g} / \mathrm{L} \mathrm{MgSO} 4 \cdot 7 \mathrm{H}_{2} \mathrm{O}, 5 \mathrm{~g} / \mathrm{L}$ yeast extract and $5 \mathrm{~mL} / \mathrm{L}$ trace metal solution.

All the culture samples $(500 \mu \mathrm{L})$ were extracted by $500 \mu \mathrm{L}$ n-butanol for three times. The combined supernatant was evaporated under vacuum and dissolved in $100 \mu \mathrm{L}$ methanol; $20 \mu \mathrm{L}$ was injected for HPLC and UPLC-MS/MS analysis. Condition of LC-MS/MS was identical to that described above.

\section{Identification and isolation of $\boldsymbol{C}$-glucosylated flavones}

In order to confirm the production of representative $C$-glycosylated flavones, we isolated three products (Api-di-C-Ara, Api-di-C-Xyl, Chr-di-C-Ara) from $1 \mathrm{~L}$ fermentation broth of strain sCZ118. The fermentation broth was all gathered by centrifuged $6000 \mathrm{rpm}$, the liquid supernatant was subjected to Diaion HP20 (Mitsubishi Co., Ltd., 1L) and eluted with increasing gradient of ethanol (from 20 to $100 \%$ ) in $\mathrm{H}_{2} \mathrm{O}$. Two fractions eluted with $60 \%$ and $80 \%$ ethanol, which contain the Api-di-CAra and minor glycosides were combined and evaporated. The residues were dissolved in 20\% methanol, subjected to MCI-gel (Mitsubishi Co., Ltd., $250 \mathrm{~mL}$ ) and eluted with increasing gradient of methanol (from 20 to $100 \%$ ) in $\mathrm{H}_{2} \mathrm{O}$. A fraction eluted with $60 \%$ methanol, which mainly contains Api-di-C-Ara was evaporated and the residue was dissolved in $10 \%$ methanol. The dissolved component was subjected to ODS silica gel column (YMC-gel ODS-A-HG, 12 nm, S-50 $\mu \mathrm{m}, 100 \mathrm{~mL}, \mathrm{YMC}$ CO., Ltd., Japan) and eluted with increasing gradient of methanol (from 10 to $100 \%$ ) in $\mathrm{H}_{2} \mathrm{O}$. Two fractions eluted with $30 \%$ and $40 \%$ methanol were was further purified repeatedly by ODS silica gel column to yield purified Apidi-C-Ara, Api-di-C-Xyl and Chr-di-C-Ara. ${ }^{1} \mathrm{H},{ }^{13} \mathrm{C}$ and 2D NMR spectra (Additional File 1: Figs. S6, S7, S8) were recorded at $80{ }^{\circ} \mathrm{C}$ on AVANCE-500 (500 MHz for $\left.{ }^{1} \mathrm{H}\right)$ spectrometer (Bruker BioSpin, Rheinstetten, Germany). The chemical shifts (ppm) were referenced to the solvent $\left(\mathrm{DMSO}-d_{6}\right)$ peaks at $\delta_{\mathrm{H}}=2.50 \mathrm{ppm}$ and $\delta_{\mathrm{C}}=39.5 \mathrm{ppm}$.

\section{Results and discussion}

\section{Rice CGTs responsible for varietal di-C-glycosides}

In our ongoing investigation of Gramineae CGTs, we first compared the differences of $C$-glycoside spectrum between two rice subspecies (japonica vs indica) in detail (Fig. 1a). The rice leaves were extracted and subjected to LC-MS/MS analysis. Because most of the flavone $C$-glycosides in rice share the common aglycone apigenin (Api) or luteolin (Lut) (Besson et al. 1985), we determined to focus on five representative groups of Api/Lut-C-glycosides, corresponding to monopentosides, monohexosides, dipentosides, pentosylhexosides and dihexosides. Both rice varieties were found to predominantly produce di-C-glycosides (96\% in O. sativa japonica, $91 \%$ in $O$. sativa indica, Fig. 1a), however, the composition of diglycosides differed drastically. The japonica rice particularly accumulated apigenin $C$-pentosylhexoside (corresponding to $m / z[\mathrm{M}-\mathrm{H}]^{-}=563.1$ ), whereas the indica rice majorly produced apigenin di-C-pentoside (corresponding to $m / z[\mathrm{M}-\mathrm{H}]^{-}=533.1$ ) besides apigenin $C$-pentosylhexoside (Fig. 1a). The most abundant diglycosides were verified as schaftoside ( $\mathrm{Sch}$ ) and apigenin 6,8-di- $C$-arabinoside (Api-di-C-Ara) as referenced to the authentic samples (Fig. 1b). In accordance with the previously recorded metabolic profiling (Kim et al. 2018a; Ramarathnam et al. 1989; Yang et al. 2016), glucosyl and arabinosyl residues seem to be the representative hexose and pentose present in rice. Other minor flavone diglycosides were proposed to be $C$-pentosylhexosides, $\mathrm{di}-C$-pentosides and di-O-glycosides with diverse sugar-linkages (Additional File 1: Fig. S2). It is also worthy to note that $C$-glycosides of apigenin are generally more abundant than those of luteolin, regardless of the glycosylation patterns (Fig. 1a).

In our previous work, both Clade A and Clade B CGTs from grass family were proved to be able to recognize the non-sugar-bearing aglycones [i.e., phloretin, 2-hydroxynaringenin (2-OHNar) (Sun et al. 2020), resulting in majorly mono- $C$-glucosides and arabinosides. It remains unclear that in rice how the aglycones undergo two steps of $C$-glycosylation to reach di- $C$-glycosides bearing different sugars (for example, schaftoside and isoschaftoside). According to the existing knowledge of flavone $C$-glycoside biosynthesis (Putkaradze et al. 2021), we proposed a biosynthetic pathway in which the rice CGTs collaborate to first install a $C$-glucosyl (mainly by Clade A CGTs) on the precursor (2-OHNar), followed by addition of a second $C$-arabinosyl group (mainly by Clade $B$ CGTs) (Fig. 2, black arrows). To verify whether Clade B CGTs could accept monoglucoside substrates like $C$-glucosyl-2-hydroxynaringenin (C-Glc-2-OHNar), we expressed the $\mathrm{His}_{6}$-tagged OsUGT708A1, OsUGT708A2, OsUGT708A39, and OsUGT708A40 in E. coli BL21(DE3) 


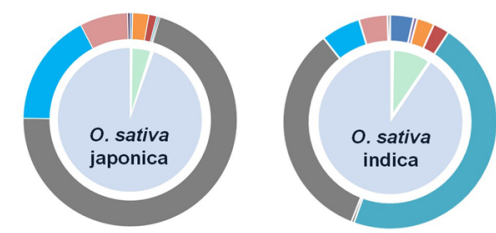

aglycone $=$ Api Lut
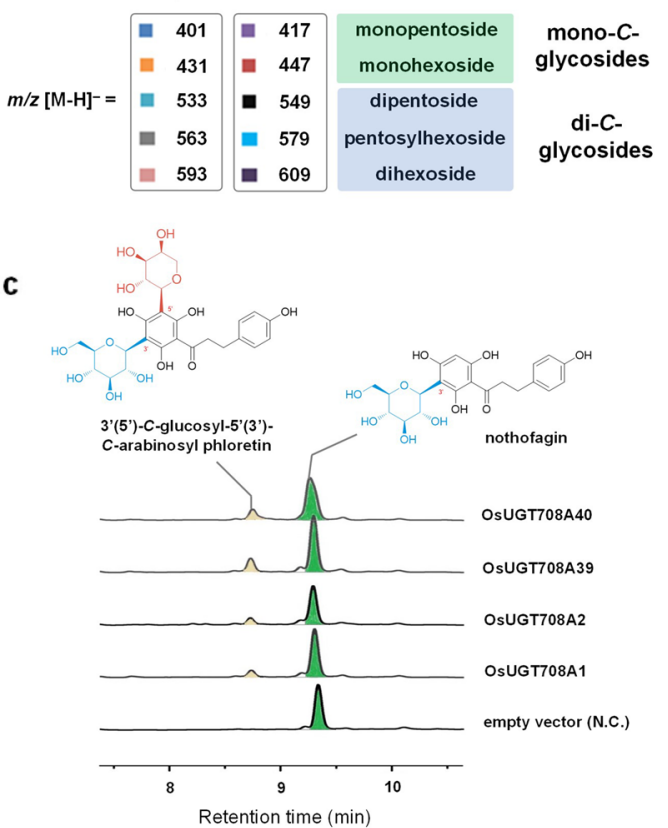

b

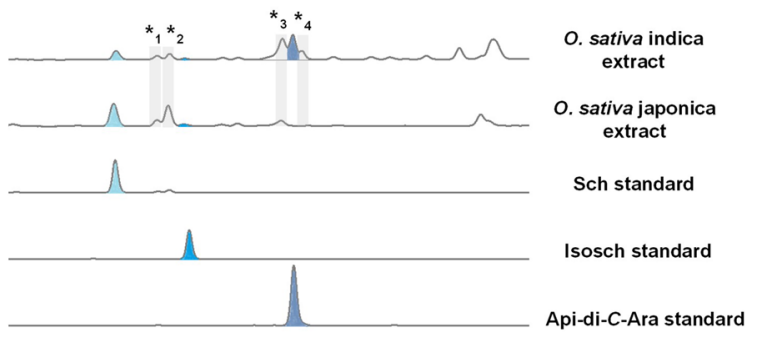

d

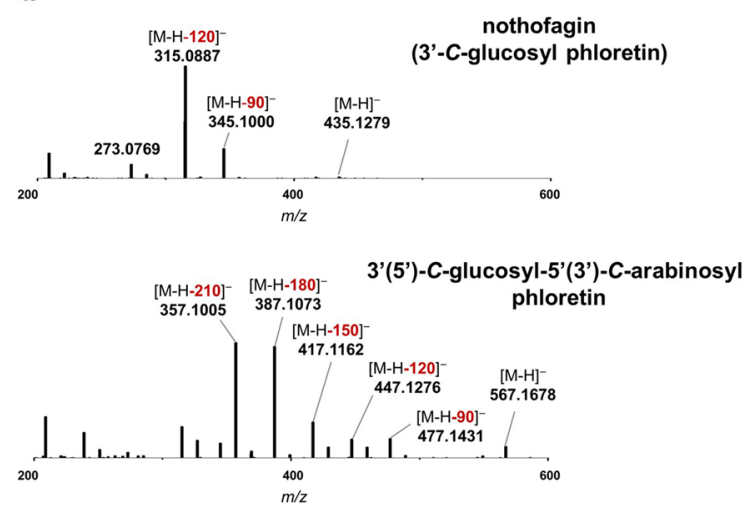

Fig. 1 Characterization of rice CGTs responsible for di-C-glycosides biosynthesis. a Composition of C-glycosides in different rice. The pie chart indicated the percentage of mono-C-glycosides and di-C-glycosides. The doughnut chart indicated individual C-glycosides of apigenin (Api) and luteolin (Lut). The ion abundance corresponding to $[\mathrm{M}-\mathrm{H}]^{-}$peaks was calculated. b HPLC analysis of rice metabolites recorded on $280 \mathrm{~nm}$. For minor diglycosides compound *1-*4, see Additional file 1: Fig S2. c HPLC chromatograms of the reactions of rice Clade B CGTs with UDP-Ara and nothofagin. $\mathbf{d}$ MS/MS fragmentation of nothofagin and its C-arabinosylated product

and tested their activities toward nothofagin $\left(3^{\prime}-C\right.$-glucosyl phloretin, a relatively stable analogue of $C$-Glc2-OHNar) in in vitro enzymatic assays. In the presence of UDP-Ara, nothofagin was converted to a new product with $m / z=567.2$ (Fig. 1c, d). The characteristic fragment ions such as $[\mathrm{M}-\mathrm{H}-150]^{-} /[\mathrm{M}-\mathrm{H}-180]^{-} /[\mathrm{M}-\mathrm{H}-210]^{-}$in MS/MS spectrum clearly revealed a hybrid pattern of $C$-pentosylation and $C$-hexosylation in good agreement with the structure of $3^{\prime}-C$-glucosyl-5' $C$-arabinosyl phloretin. These results suggested that rice Clade B CGTs could catalyze the arabinosyl-transferring reaction of $C$-monoglucoside substrate, which was a key step in the biosynthesis of $C$-pentosylhexosides like schaftoside or isoschaftoside. Recently, the dissection of schaftoside pathway in other plants (mainly represented by dicot plants like Scutellaria baicalensis and Nelumbo nucifera) also supported the above results (Feng et al. 2021; Wang et al. 2020), which shows the generality of di-C-glycoside pathway in higher plants.

Unlike $O$. sativa japonica that accumulates $C$-pentosylhexoside, $O$. sativa indica produces a large amount of apigenin di-C-pentoside occupying $46 \%$ of the total flavone $C$-glycosides (Fig. 1a). There is also an increase of apigenin mono- $C$-pentoside (corresponding to $\mathrm{m} / \mathrm{z}$ $\left.[\mathrm{M}-\mathrm{H}]^{-}=401.1\right)$. This is probably due to the three additional clade B CGTs (OsUGT708A1, OsUGT708A39 and OsUGT708A40) only present in indica rice (Fig. 1), which can utilize UDP-Ara to convert 2-OHNar to C-Ara-2-OHNar (alternatively, phloretin to $C$-arabinosyl phloretin) (Sun et al. 2020). In particular, among the Clade B CGTs, OsUGT708A40 is a unique di- $C$-arabinosyltransferase that catalyzes a tandem $C$-arabinosylation reaction (Sun et al. 2020). We proposed that OsUGT708A40 was a key di-C-arbinosyltransferase 
a

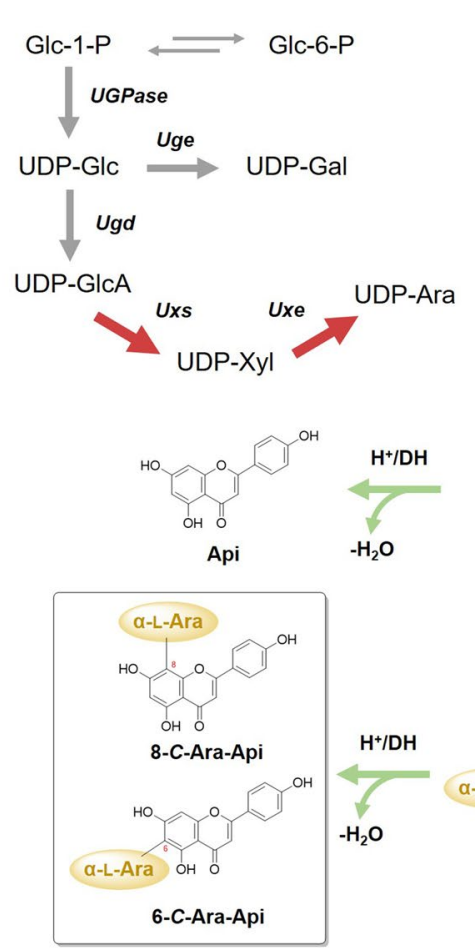

b

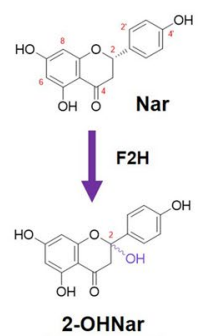

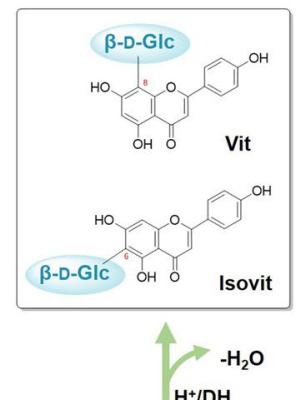

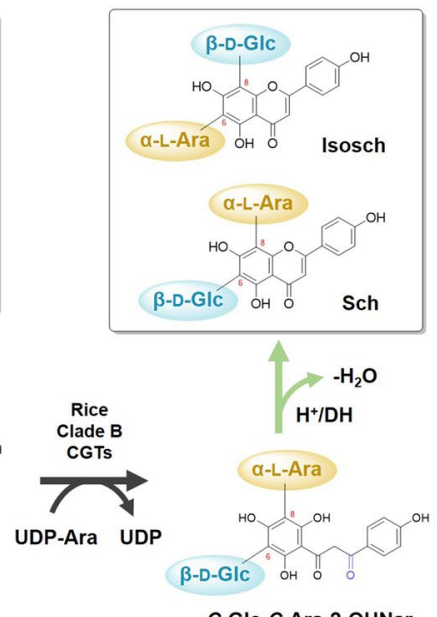

C-GIC-2-OHNar (open ring form)

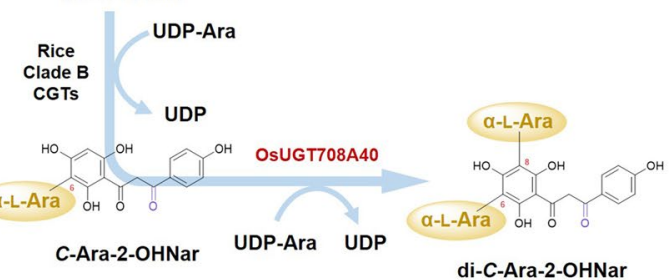

di-C-Ara-2-OHNar

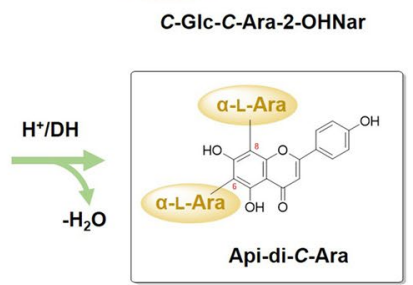

Fig. 2 A proposed biosynthetic network of C-glycosylated apigenin in rice. a UDP-arabinose (Ara) is generated from glucose-1-phosphate (Glc-1-P) via UDP-glucose (Glc) and UDP-xylose (Xyl). Grey arrows represent the native metabolism in E. coli, while the bold red arrows represent an introduced heterologous UDP-Ara pathway. Glc-6-P glucose-6-phosphate, Gal galactose, GlcA glucuronate, UGPase UDP-glucose pyrophosphorylase, Uge UDP-glucose 4-epimerase, Ugd UDP-glucose 6-dehydrogenase, Uxs UDP-xylose synthase/UDP-glucuronic acid decarboxylase, Uxe UDP-xylose 4-epimerase. b A proposed C-glycosylated apigenin pathway starting from naringenin (Nar). Purple arrows represent 2-hydroxylation by flavanone 2-hydroxylases ( $F 2 \mathrm{H}$ ) and equilibrium of ring-open/closed 2-hydroxynaringenin (2-OHNar). Black arrows indicate two-step glycosylation reactions in (iso)schaftoside biosynthesis ( $\mathrm{Sch}$ ). Glycosylation reactions forming apigenin 6,8-di-C-arabinoside (Api-di-C-Ara) are indicated in sky blue arrows. After the formation of C-glycosylated intermediates, dehydration reactions (green arrows) occur spontaneously in acidic solvent or by dehydratases $(\mathrm{DH})$, producing a mixture of 6-C- or 8-C-isomers. Api apigenin, 2OHNar 2-hydroxylnaringenin, Vit vitexin, Isovit isovitexin

responsible for the formation of apigenin 6,8-di-C-arabinoside (Fig. 2, light blue arrows).

\section{Introduction of UDP-arabinose and UDP-xylose supply allowed de novo biosynthesis of schaftoside, isoschaftoside, vicenin- 1 and vicenin-3}

To further prove our proposed pathway (Fig. 2a, b) and achieve de novo biosynthesis of bioactive di-C-glycosides, we selected the fast growing and genetically amenable $E$. coli as a suitable chassis for pathway reconstitution. The previously constructed sCZ112 harboring pYH55 (Li et al. 2019) and pCZ201 (Sun et al. 2020) for optimized 2-hydroxynaringenin production was used as the starting strain. In order to realize the heterologous biosynthesis of $C$-pentosylhexoside like schaftoside, we first assembled a di-CGT cassette containing PhUGT708A43 (an excellent coding C-monoglucosylating enzyme from moso bamboo (Sun et al. 2020) for the first step of glucosylation) and OsUGT708A1 (for the subsequent $C$-arabinosylation) under $\mathrm{T} 7$ promoter (Fig. 3a).

A major difficulty for the biosynthesis of arabinosides in E. coli is the absence of native UDP-arabinose supply. To solve this problem, we introduced SmUxs (UDPxylose synthase) and SmUxe (UDP-xylose 4-epimerase) from Sinorhizobium meliloti 1021 (Gu et al. 2011) to enable the metabolism from UDP-glucose to UDP-arabinose (Fig. 2a). Two SmUxs homologues (SmUxs1 and SmUxs2), sharing only $57.3 \%$ amino acid identity, were, respectively, ligated downstream to the PhUGT708A43OsUGT708A1 cassette and further assembled with SmUxe to give pCZ193-1 and pCZ193-2 ready for the production of schaftoside (Fig. 3a). After transferring pCZ193-1 or pCZ193-2 into sCZ112 (resulting in strain sCZ113 and sCZ114, respectively), we successfully detected $2.75 \mathrm{mg} / \mathrm{L}$ schaftoside (Sch) and $0.43 \mathrm{mg} / \mathrm{L}$ 
a

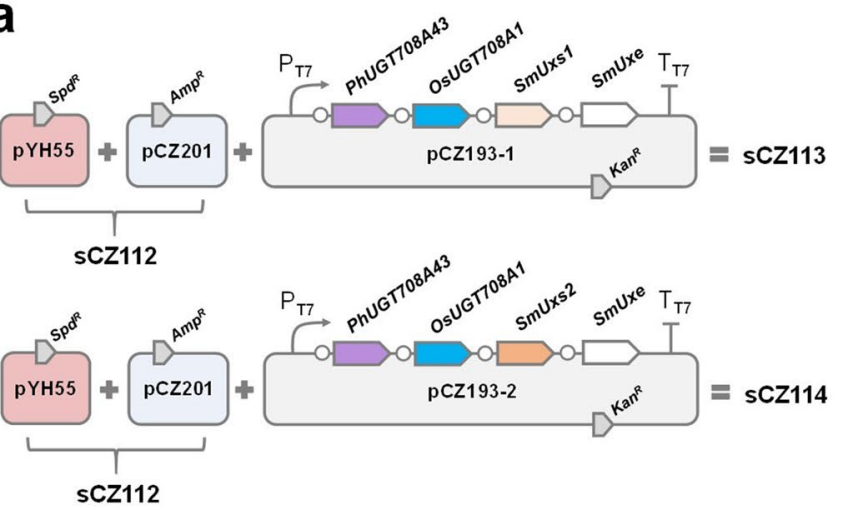

$\mathrm{P}_{\mathrm{T} 7}=\mathrm{T} 7$ promotor $\quad \mathrm{T}_{\mathrm{T} 7}=\mathrm{T} 7$ terminator $\mathrm{O}=$ ribosome binding site (RBS)

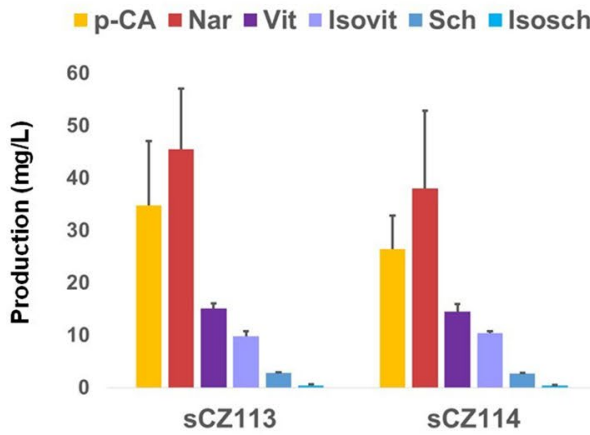

b

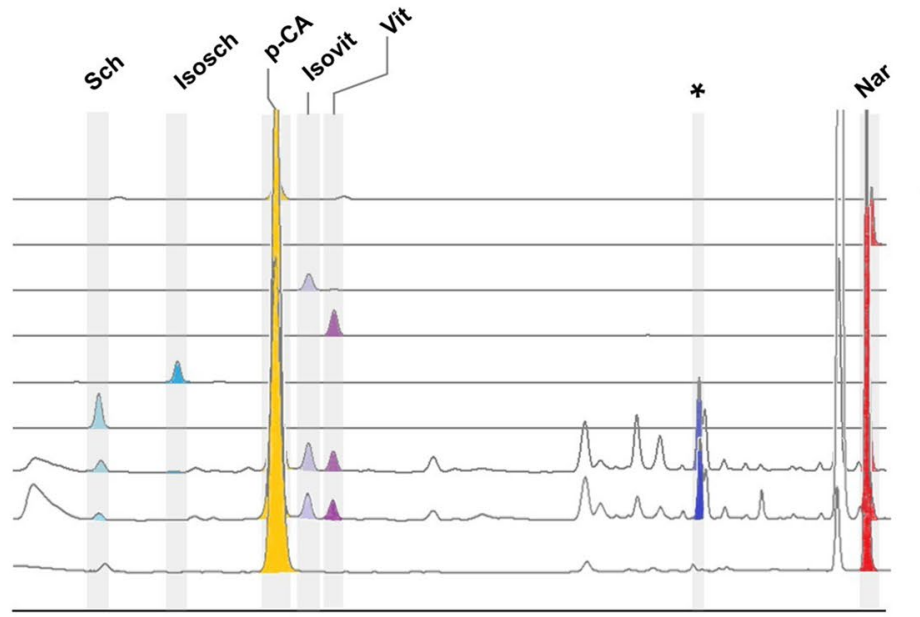

p-coumaric acid standard naringenin standard

isovitexin standard

vitexin standard

schaftoside standard

isoschaftoside standard

sCZ113 extract

sCZ114 extract

sCZ110 extract (negative control)

15

20
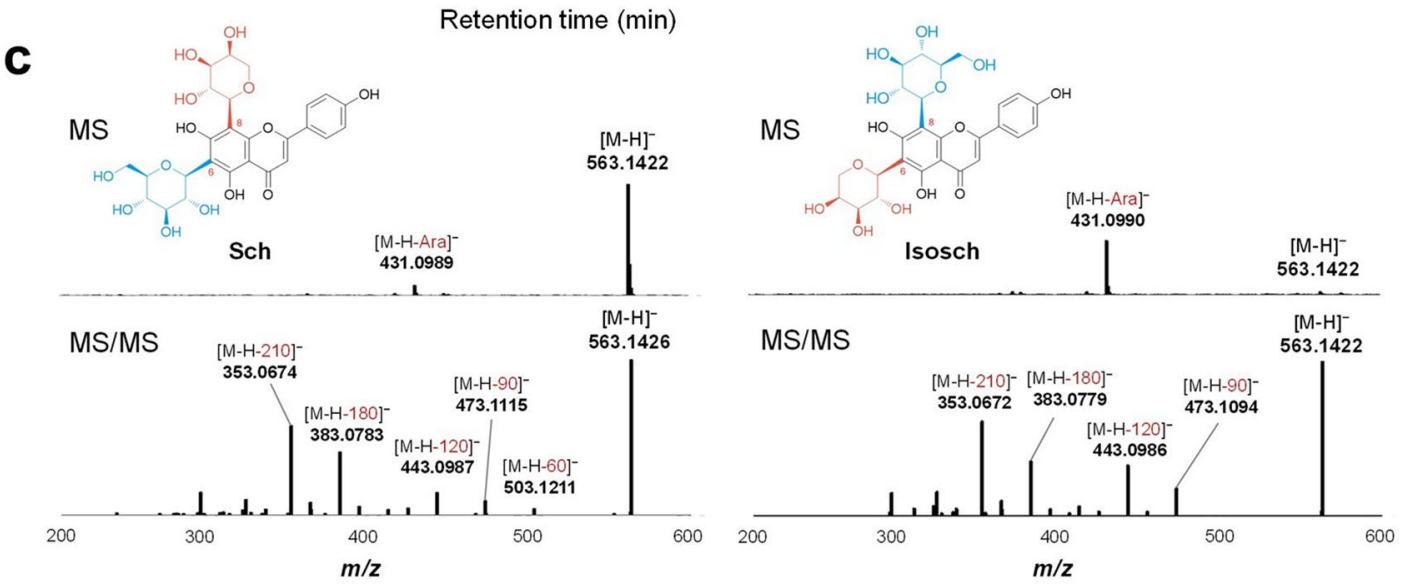

Fig. 3 De novo biosynthesis of schaftoside. a Reconstitution of schaftoside pathway in E. coli chases. pYH55 (Li et al. 2019) is assembled for naringenin (Nar) production and pCZ201 (Sun et al. 2020) harbors cytochrome P450 module for 2-hydroxylnaringenin (2-OHNar) production. Fermentation of sCZ113 and sCZ114 revealed similar productivity. $\mathbf{b}$ HPLC chromatography of the extract of sCZ113. Standard samples were also analyzed for comparison. The peak indicated in asterisk was temporarily identified as apigenin 6(8)-C-arabinoside. UV absorbance at $280 \mathrm{~nm}$ was monitored. (C) MS and MS/MS spectra of schaftoside (Sch) and isoschaftoside (Isosch) present in the extract of sCZ113 
isoschaftoside (Isosch) in sCZ113 broth through 72-h fermentation in MOPS media (Fig. 3b). The pathway intermediates like vitexin (Vit, $15.14 \mathrm{mg} / \mathrm{L}$ ), isovitexin (Isovit, $9.78 \mathrm{mg} / \mathrm{L}$ ), naringenin (Nar, $45.54 \mathrm{mg} / \mathrm{L}$ ) and $p$-coumaric acid (p-CA, $34.79 \mathrm{mg} / \mathrm{L}$ ) were also observed (Fig. 3a, b). All the products were identified through comparison with authentic samples in HPLC analysis (Fig. 3b) and high-resolution (HR) MS/MS spectroscopic data (Fig. 3c, Additional File 1: Fig. S3). On the other hand, $2.67 \mathrm{mg} / \mathrm{L}$ Sch and $0.41 \mathrm{mg} / \mathrm{L}$ Isosch were detected in sCZ114. The accumulation of Vit, Isovit and Nar reached $14.52 \mathrm{mg} / \mathrm{L}$, $10.42 \mathrm{mg} / \mathrm{L}$ and $38.01 \mathrm{mg} / \mathrm{L}$. A similar productivity of Sch/Isosch and no significant difference of accumulation pattern of intermediates between SmUxs1 and SmUxs2 (Fig. 3a), therefore we used SmUxs1 for further experiments.

Since UDP-xylose is an upstream precursor of UDParabinose (Fig. 2a), we proposed that flavone $C$-xylosides might be generated in a truncated pathway containing biosynthetic genes fitting just for UDP-xylose biosynthesis (Additional File 1: Fig. S4). Therefore, we also try to achieve the production of vicenin-1 (apigenin 6-C-xylosyl-8-C-glucoside, Vic-1) and vicenin-3 (apigenin 6-C-glucosyl-8- $C$-xyloside, Vic-3). After transferring pCZ192-1 (harbors the cassette of PhUGT708A43-OsUGT708A1-SmUxs1) into sCZ112 (resulting in strain sCZ115), we detected a trace amount of Vic-1 $(0.09 \mathrm{mg} / \mathrm{L})$ and Vic-3 $(0.28 \mathrm{mg} / \mathrm{L})$ in $72 \mathrm{~h}$ fermentation (Additional File 1: Fig. S5), which is a much lower titer compared to that of Sch and Isosch. This result indicated that UDP-xylose might not be a favorite sugar donor of OsUGT708A1. To the best of our knowledge, di-C-glycosides like Sch, Isosch, Vic-1 and Vic-3 were synthesized in heterologous chassis cells for the first time. These results indicated the feasibility of de novo production of $C$-arabinoside and $C$-xyloside in $E$. coli.

\section{De novo biosynthesis of apigenin di-C-arabinoside and minor C-pentosides}

Flavone compounds bearing multiple $C$-pentosyl (for example, arabinosyl, xylosyl) residues are uncommon natural products. To further expand the diversity of flavone $C$-glycosides, we attempted to construct an artificial pathway in E. coli for the production of apigenin di- $C$-arabinoside and other minor $C$-pentosides. The biosynthesis of specific di- $C$-arabinosides requires efficient di-C-glycosyltransferase preferring UDP-Ara, as well as a heterologous UDP-Ara-synthesizing module above-mentioned. OsUGT708A40 was selected as a proper enzymatic part since it was identified as the only di- $C$-arabinosyltransferase in rice (Sun et al. 2020). Due to the close similarity of UDP-Ara and UDP-xylose
(UDP-Xyl), we predicted that OsUGT708A40 might also promiscuously consume UDP-Xyl for some minor $C$-xyloside production.

The construct pCZ194 harboring OsUGT708A40SmUxs1-SmUxe cassette was transformed into sCZ112 to give strain sCZ118 (Fig. 4a). After $72 \mathrm{~h}$ fermentation, we detected the emergence of new products characteristic of $C$-pentosides instead of $C$-glucosides (i.e., Vit/Isovit) in the fermentation media. We quantified these products using vitexin and vicenin-2 as internal standards. The major peak with a retention time $\left(R_{t}\right)=16.02 \mathrm{~min}$ was identified as apigenin 6,8-C-di-arabinoside (Api-di$C$-Ara) based on both LC-MS/MS and NMR evidence (Fig. 4b, Additional File 1: Fig. S6). The ${ }^{1} \mathrm{H}$ NMR spectrum of Api-di-C-Ara recorded at $80{ }^{\circ} \mathrm{C}$ clearly revealed the doublets of aglycone $\mathrm{H} 2, \mathrm{6}^{\prime}$ and two anomeric protons of sugars (Additional File 1: Fig. S7). The $\alpha$-L-arabinosyl was clarified though correlations between Ara-H1, Ara$\mathrm{H} 5 \mathrm{a}$ and Ara-H4 observed in 2D NOESY spectrum (Additional File 1Fig. S6f). In addition to Api-di-C-Ara, we also detected two minor $C$-pentosides which are supposed to be apigenin 6,8-C-di-xyloside (Api-di- $C$ $\mathrm{Xyl}$ ) and chrysin 6,8-C-di-arabinoside (Chr-di-C-Ara), according to the HR-MS/MS and NMR analyses (Fig. 4c, Additional File 1: Fig. S8). Unfortunately, we didn't succeed in obtaining and distinguishing apigenin 6-C-arabinoside or 8-C-arabinoside [Api-(6/8)-C-Ara], since they decomposed quickly in the solvent (Additional File 1: Fig. S9). Without extra optimization, the titer of Api-di-CAra, Api-di-C-Xyl, Chr-di-C-Ara and nascent Api-C-Ara reached $24.89 \mathrm{mg} / \mathrm{L}, 0.78 \mathrm{mg} / \mathrm{L}, 0.38 \mathrm{mg} / \mathrm{L}, 21.15 \mathrm{mg} / \mathrm{L}$, respectively.

Similarly, we also constructed pCZ195 specific for Api-di-C-Xyl production (Additional File 1: Fig. S8). As expected, after $72 \mathrm{~h}$ fermentation, we detected $3.26 \mathrm{mg} / \mathrm{L}$ Api-di-C-Xyl as major product with no flavone $C$-arabinosides accumulated (Additional File 1: Fig. S10). While compared with the productivity of Api-di-C-Ara $(24.89 \mathrm{mg} / \mathrm{L})$ in sCZ118, the production of Api-di-C-Xyl was much lower. This could also be explained by the substrate preference of OsUGT708A40 to UDP-Ara rather than to UDP-Xyl.

\section{Fed-batch fermentation of $C$-arabinosides}

To achieve a large-scale production and verify the scalability of our $C$-glycoside-producing strains, we performed scale-up fermentation of sCZ113 and sCZ118 in a 5-L bioreactor. The minimal M9 media with $20 \mathrm{~g} / \mathrm{L}$ glucose was used as basal culture medium and $500 \mathrm{~g} / \mathrm{L}$ glucose was used as supplementary medium. During the fermentation process of sCZ113, $p$-coumaric acid (p-CA) rapidly accumulated to $66.1 \mathrm{mg} / \mathrm{L}$ at $9 \mathrm{~h}$ (after induction) at the first stage and then rapidly decreased (Fig. 5a). 


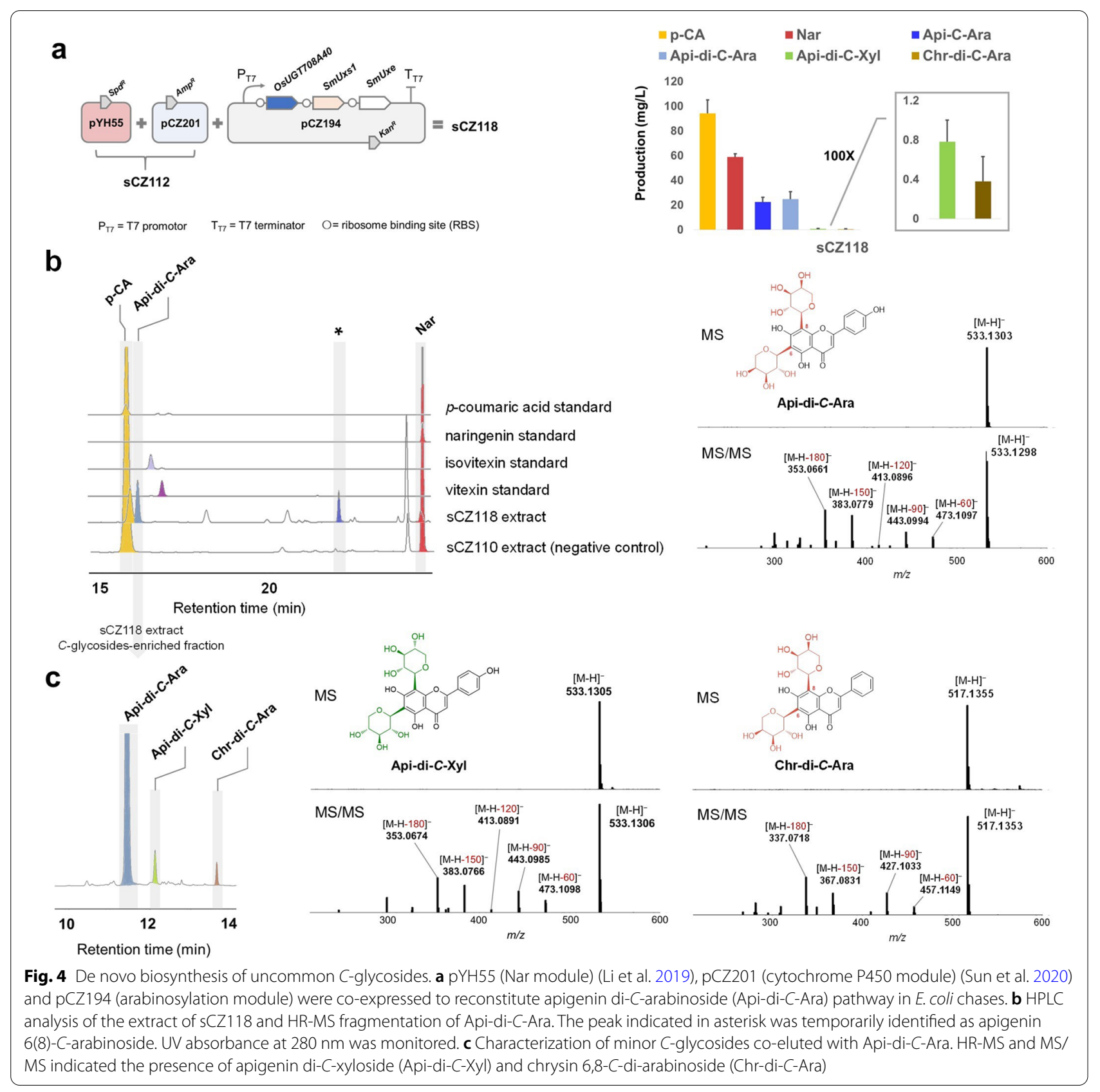

Afterwards, naringenin (Nar) accumulated to $75.8 \mathrm{mg} / \mathrm{L}$ at $16 \mathrm{~h}$ until it was consumed. Vitexin (Vit) and isovitexin (Isovit) appeared at about $9 \mathrm{~h}$, and schaftoside (Sch)/ isoschaftoside (Isosch) appeared later (at approximate $16 \mathrm{~h}$ ). After $81 \mathrm{~h}$ fermentation, production of Sch and Isosch reached $19.87 \mathrm{mg} / \mathrm{L}$ (7.2-fold compared to flaskshake) and $2.41 \mathrm{mg} / \mathrm{L}$ (5.6-fold compared to flask-shake) with $22.87 \mathrm{mg} / \mathrm{L}$ Vit and $13.32 \mathrm{mg} / \mathrm{L}$ Isovit left.

During the fermentation process of sCZ118, p-CA $(74.8 \mathrm{mg} / \mathrm{L})$ and Nar $(20.29 \mathrm{mg} / \mathrm{L})$ first rapidly accumulated to the maximum within $9 \mathrm{~h}$ (Fig. 5b). After $84 \mathrm{~h}$ fermentation, production of Api-di-C-Ara reached to $113.16 \mathrm{mg} / \mathrm{L}$ (4.7-fold compared to flask-shake). The results confirm that our fermentation process could be scaled up controllably and productively, which proved that fed-batch fermentation was beneficial to the accumulation of downstream glycosylated products. Our engineered $E$. coli system has the ability to supply enough UDP-Ara for large production of flavone $C$-arabinosides, which displays great industrial potential. 

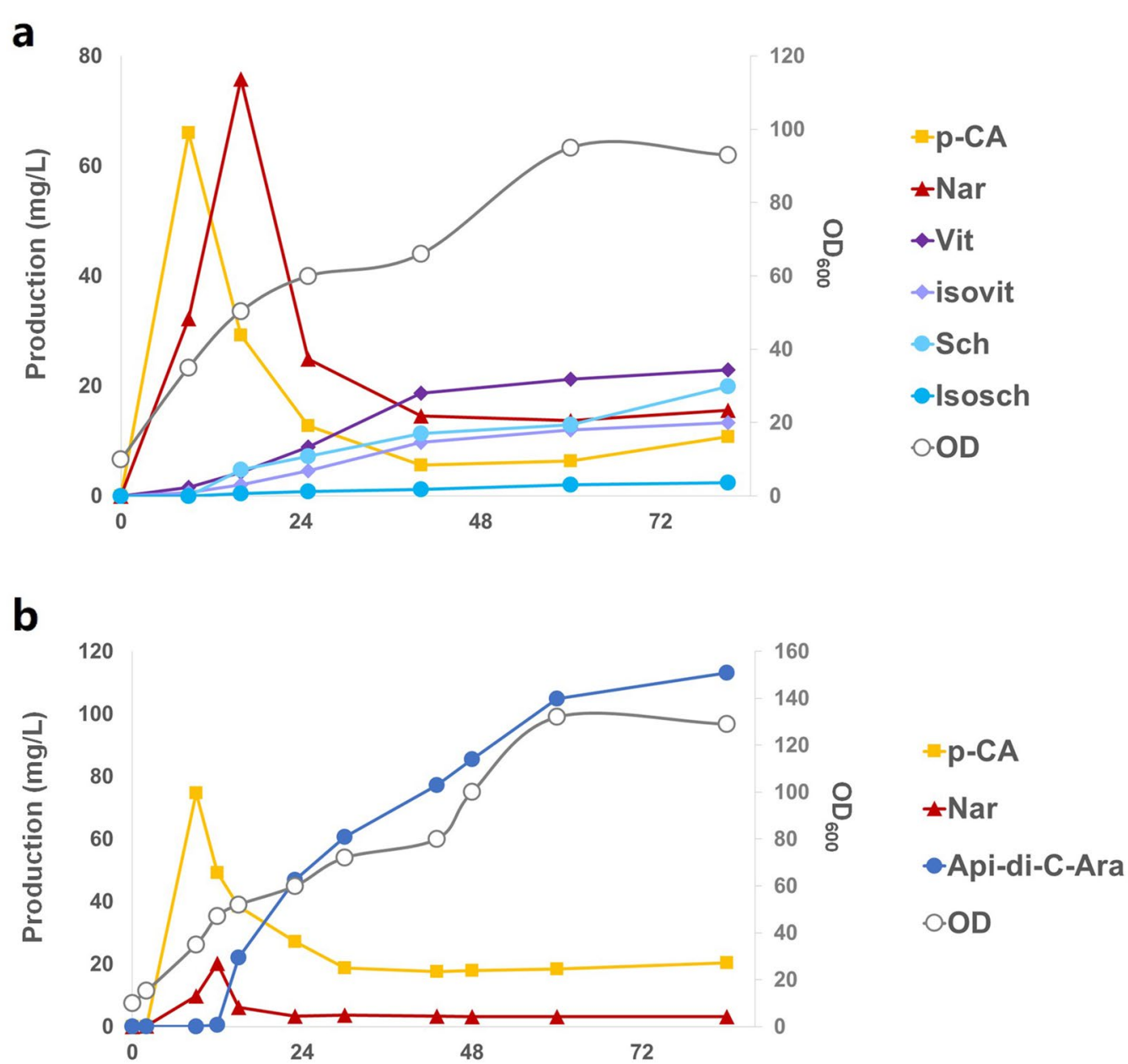

Fig. 5 Fed-batch fermentation of C-arabinosides. a Fed-batch fermentation of sCZ113 in 5-L bioreactor. b Fed-batch fermentation of sCZ118 in 5-L bioreactor

\section{Conclusion}

Rice (Oryza sativa) is one of the most important crops feeding more than 3 billion of people. The subspecies indica and japonica are two main varieties of the cultivated rice. Investigation of the difference between two close subspecies has always been an interesting topic. In this research, we discovered dramatic difference of the $C$-glycosylated flavones, especially the metabolites containing arabinosyls occurring in two rice subspecies. Schaftoside featuring a hybrid $C$-glucosylation/ $C$-arabinosylation is the most abundant diglycoside metabolite in japonica rice. In our previous work, japonica rice-originated OsUGT708A2, OsUGT708A3 and OsUGT708A4 were all identified as $C$-glucosyltransferases acting on aglycone substrates (phloretin, 2OH-Nar). Through the analyses of enzymatic function, we demonstrated this time that OsUGT708A2 (belongs to Clade B) was also able to $C$-arabinosylate monoglucoside substrates, which might explain the formation of flavone $C$-pentosylhexosides like schaftoside and isoschaftoside. This result is in good agreement with the recent work reported by (Wang et al. 2020). Due to the absence of other mono- and di$C$-arabinosyltransferases in japonica rice, mono- and di- $C$-arabinoside was barely detected. In comparison, $O$. sativa indica produces apigenin $\mathrm{di}-\mathrm{C}$-arabinoside as the major flavone $C$-glycoside. We proposed that the specific CGTs in indica rice (OsUGT708A1, OsUGT708A39 and OsUGT708A40) influenced the accumulation pattern of flavone $C$-glycosides and caused diverse metabolisms in different rice cultivars. In particular, OsUGT70A40 may catalyze tandem $C$-arabinosylation to form di- $C$-arabinoside. Such different metabolic profiling was also observed in minor products of rice, as japonica rice accumulated more chrysoeriol $C$-glucosyl- $C$-arabinoside (compound *2) than indica rice did, while chrysoeriol di-C-arabinoside (compound *4) was only found in indica rice 
(Fig. 1b and Additional File 1: Fig. S2). Overall, hybrid $C$-glucosylation/ $C$-arabinosylation is more common in japonica rice and di- $C$-arabinosylation is the major flavone decoration in indica rice. The expansion of rice clade B CGTs represents a good example of how plants evolve new enzymes to diversify their particular chemicals, suggesting the importance of $C$-glycosyltransferases in plant metabolism.

In nature, the grass family plants produce a highly complex mixture of $C$-glycosides consisting of $C$-pentosylhexoside, mono- $C$ - and di- $C$-pentosides. It is timeconsuming to isolate and purify these compounds, which perhaps hinders the evaluation of their potential pharmaceutical and nutraceutical values. Due to the rarity of $C$-arabinosyl-transferring bio-parts and the expensiveness of UDP-arabinose and UDP-xylose, there has been no report on the de novo heterologous biosynthesis of $C$-arabinoside and $C$-xylose in microorganism chassis up to now. Through integration of all genes involved in the flavone $C$-arabinosides and flavone $C$-xylose pathway and introduction of UDP-arabinose and UDP-xylose biosynthesis genes, de novo synthesis of several flavone $C$-arabinosides was preliminarily realized in our engineered E. coli strains. Moreover, through high-density fed-batch fermentation, we achieved a high titer of several desired $C$-arabinosides and $C$-xylosides, which proved the feasibility of E. coli strains as platform for production of flavone $C$-arabinosides and $C$-xylosides. Unexpectedly, in the fermentation of sCZ113 and sCZ114, the production of isoschaftoside was much lower than schaftoside. This may be due to endogenous dehydratase, yet not identified, preferentially eliminating 2-hydroxyls of 2-OHNar to give a 6 - $C$-glucosyl-8- $C$-arabinosyl isomer. This phenomenon is particular because $6-C$ - and $8-C$ - mixture is always observed in the reported work of de novo biosynthesis of $C$-monoglucoside (Vanegas et al. 2018; Sun et al. 2020).

The production of minor product chrysin $6,8-C$-diarabinoside was proposed to rise from the promiscuity of tyrosine ammonia lyase (TAL) in pYH55, which recognizes both L-tyrosine and L-phenylalanine as precursors ( $\mathrm{Li}$ et al. 2019). In addition, significant discrepancy of the productivity between $C$-arabinosides and Api$C$-xylosides in our constructed strains again supported that UDP-Ara was preferred. This preference of $C$-glycosyltransferases leads to the difference of $C$-glycoside metabolite contents in different rice, which highlighted synthetic biology as more meaningful approach for largescale manufacturing of rare natural product through the utilization of specific $C$-glycosyltransferases.

E. coli does not possess the ability to synthesize UDP-Ara and UDP-Xyl. Introducing an exogenous
UDP-Ara and UDP-Xyl biosynthetic pathway to achieve a high production of $C$-glycoside adequately indicated the potential of wider application prospect. Some relevant approaches such as strengthening UDPGlc supply and replacing Uxs and Uxe from other species will both bring benefits to this pathway. Also, by modification of the $C$ terminal of known CGTs, catalytic pocket mores suitable for UDP-Xyl recognition could be designed, helping engineered strain to reach a higher production of $C$-xylosides. Further study could be focused on downstream products of diglycosides, such as carlinoside, isocarlinoside, lucenin- 1 and lucenin-3 if the corresponding flavone $3^{\prime}$-hydroxylase $\left(\mathrm{F}^{\prime} \mathrm{H}\right)$ is further incorporated. E. coli platform and synthetic biology will become great assist to the development of flavone $C$-arabinosides.

\section{Abbreviations}

UDP-Glc: UDP-glucose; UDP-Ara: UDP-arabinose; UDP-Xyl: UDP-xylose; Sch: Schaftoside; Isosch: Isoschaftoside; Vic-1: Vicenin-1; Vic-3: Vicenin-3; Phr: Phloretin; Vit: Vitexin; Isovit: Isovitexin; Nar: Naringenin; p-CA: $p$-Coumaric acid; Api-di-C-Ara: Apigenin 6,8-C-di-arabinoside; Api-di-C-Xyl: Apigenin 6,8-C-dixyloside; 2-OHNar: 2-Hydroxynaringin.

\section{Supplementary Information}

The online version contains supplementary material available at https://doi. org/10.1186/s40643-021-00404-3.

Additional file1: Fig. S1. An unrooted phylogenetic tree of rice CGTs. Fig S2. LC-MS/MS analyses of minor flavone glycosides present in O. sativa. Fig. S3. LC-MS/MS analyses of pathway intermediates in the extracts of sCZ113 and sCZ118. Fig. S4. A proposed biosynthetic network of flavone C-xylosides. Fig. S5. De novo biosynthesis of vicenin-3 and vicenin-1. Fig. S6. NMR spectra of apigenin 6,8-C-di-arabinoside (Api-di-C-Ara). Fig. S7. Comparison of $1 \mathrm{H}$ NMR spectra of Api-di-C-Ara recorded at different temperature (K). Fig. S8. 1 H NMR spectra of (A) apigenin 6,8-di-C-xyloside (Api-di-C-Xyl) and (B) chrysin 6,8-di-C-arabinoside (Chr-di-C-Ara) recorded at 353 K. Fig. S9. Decomposition of apigenin mono-C-arabinosides. Fig. S10. De novo biosynthesis of Api-di-C-Xyl. Table S1. Plasmids and strains used in this study. Table S2. Primers used in this study.

\section{Acknowledgements}

We would like to thank Xiaoyan Xu, Shizhen Bu in the Core Facility Centre of SIPPE for technical assistance on HR-MS/MS and NMR acquisition.

\section{Authors' contributions}

YW, YS and ZC designed the study. ZC and YS performed the main part of experiments and wrote the manuscript. GW isolated and characterized the compounds. Yi Z, QZ, Yu Z and JL contributed to enzyme study and data analysis. All authors contributed to discussion of the manuscript. All authors have read and approved the final manuscript.

\section{Funding}

This study was funded from the National Key R\&D Program of China (2018YFA0900600), the Program of Shanghai Academic Research Leader (20XD1404400), the Strategic Priority Research Program "Molecular mechanism of Plant Growth and Development" of CAS (XDB27020202), the National Natural Science Foundation of China (Grant nos. 32070328, 41876084, 22077129), the Construction of the Registry and Database of Bioparts for Synthetic Biology of the Chinese Academy of Science (No. ZSYS-016), the International Partnership Program of Chinese Academy of Science (No. 
153D31KYSB20170121) and the National Key Laboratory of Plant Molecular Genetics, SIPPE, CAS. Y. S. is supported by the Foundation of Youth Innovation Promotion Association of the Chinese Academy of Sciences.

\section{Availability of data and materials}

All data generated or analyzed during this study are included in this published article (and its supplementary information files).

\section{Declarations}

\section{Ethics approval and consent to participate}

Not applicable.

\section{Consent for publication}

Not applicable.

\section{Competing interests}

The authors declare that they have no competing interests.

\section{Author details}

${ }^{1}$ CAS-Key Laboratory of Synthetic Biology, CAS Center for Excellence in Molecular Plant Sciences, Institute of Plant Physiology and Ecology, Chinese Academy of Sciences, Shanghai 200032, China. ${ }^{2}$ University of Chinese Academy of Sciences, Beijing 100039, China.

Received: 25 March 2021 Accepted: 7 June 2021 Published online: 12 June 2021

\section{References}

Besson E, Dellamonica G, Chopin J, Markham KR, Kim M, Koh H, Fukami H (1985) C-glycosylflavones from Oryza sativa. Phytochemistry 24:10611064. https://doi.org/10.1016/S0031-9422(00)83183-0

Brazier-Hicks M, Edwards R (2013) Metabolic engineering of the flavoneC-glycoside pathway using polyprotein technology. Metab Eng 16:11-20. https://doi.org/10.1016/j.ymben.2012.11.004

Chen D, Fan S, Chen R, Xie K, Yin S, Sun L, Liu J, Yang L, Kong J, Yang Z, Dai J (2018) Probing and engineering key residues for bis-C-glycosylation and promiscuity of a C-glycosyltransferase. ACS Catal 8:4917-4927. https:// doi.org/10.1021/acscatal.8b00376

Das S, Teja KC, Mukherjee S, Seal S, Sah RK, Duary B, Kim K, Bhattacharya SS (2018) Impact of edaphic factors and nutrient management on the hepatoprotective efficiency of carlinoside purified from pigeon pea leaves: An evaluation of UGT1A1 activity in hepatitis induced organelles. Environ Res 161:512-523. https://doi.org/10.1016/j.envres.2017.11.054

Ettayapuram Ramaprasad AS, Durkin KA, Smith MT (2020) Structure-based virtual screening of a natural product database to identify several possible SARS-CoV-2 main protease inhibitors. ChemRxiv. https://doi.org/10. 26434/chemrxiv.12143394

Feng C, Li S, Taguchi G, Wu Q, Yin D, Gu Z, Wu J, Xu W, Liu C, Wang L (2021) Enzymatic basis for stepwise C-glycosylation in the formation of flavonoid di-C-glycosides in sacred lotus (Nelumbo nucifera Gaertn.). Plant J 106:351-365. https://doi.org/10.1111/tpj.15168

Gu X, Lee S, Bar-Peled M (2011) Biosynthesis of UDP-xylose and UDP-arabinose in Sinorhizobium meliloti 1021: first characterization of a bacterial UDPxylose synthase, and UDP-xylose 4-epimerase. Microbiol 157:260-269. https://doi.org/10.1099/mic.0.040758-0

He J, Zhao P, Hu Z, Liu S, Kuang Y, Zhang M, Li B, Yun C, Qiao X, Ye M (2019) Molecular and structural characterization of a promiscuous C-glycosyltransferase from Trollius chinensis. Angew Chem Int Ed 58:11513-11520. https://doi.org/10.1002/anie.201905505

Hooper AM, Tsanuo MK, Chamberlain K, Tittcomb K, Scholes J, Hassanali A, Khan ZR, Pickett JA (2010) Isoschaftoside, a C-glycosylflavonoid from Desmodium uncinatum root exudate, is an allelochemical against the development of Striga. Phytochemistry 71:904-908. https://doi.org/10. 1016/j.phytochem.2010.02.015

Ito T, Fujimoto S, Shimosaka M, Taguchi G (2014) Production of C-glucosides of flavonoids and related compounds by Escherichia coli expressing buckwheat C-glucosyltransferase. Plant Biotechnol 31:519-524. https:// doi.org/10.5511/plantbiotechnology.14.1016a

Joshi T, Joshi T, Sharma P, Mathpal S, Pundir H, Bhatt V, Chandra S (2020) In silico screening of natural compounds against COVID-19 by targeting Mpro and ACE2 using molecular docking. Eur Rev Med Pharmacol Sci 24:4529-4536. https://doi.org/10.26355/eurrev_202004_21036

Kim S, Lee H, Park K, Kim B, Ahn J (2015) Metabolic engineering of Escherichia coli for the biosynthesis of flavonoid-O-glucuronides and flavonoidO-galactoside. Appl Microbiol Biotechnol 99:2233-2242. https://doi.org/ 10.1007/s00253-014-6282-6

Kim B, Woo S, Kim M, Kwon S, Lee J, Sung S, Koh H (2018a) Identification and quantification of flavonoids in yellow grain mutant of rice (Oryza sativa L.). Food Chem 241:154-162. https://doi.org/10.1016/j.foodchem.2017. 08.089

Kim P, Shin J, Jo D, Shin D, Choi D, Kim W, Park K, Kim J, Joo C, Lee J, Choi Y, Shin Y, Shin J, Jeon H, Seo J, Cho D (2018b) Anti-melanogenic activity of schaftoside in Rhizoma Arisaematis by increasing autophagy in B16F1 cells. Biochem Biophys Res Commun 503:309-315. https://doi.org/10. 1016/j.bbrc.2018.06.021

Li J, Tian C, Xia Y, Mutanda I, Wang K, Wang Y (2019) Production of plantspecific flavones baicalein and scutellarein in an engineered $E$. coli from available phenylalanine and tyrosine. Metab Eng 52:124-133. https://doi. org/10.1016/j.ymben.2018.11.008

Lim C, Wong L, Bhan N, Dvora H, Xu P, Venkiteswaran S, Koffas MA (2015) Development of a recombinant Escherichia coli strain for overproduction of the plant pigment anthocyanin. Appl Environ Microbiol 81:6276-6284. https://doi.org/10.1128/AEM.01448-15

Liu M, Liu C, Chen H, Huang X, Zeng X, Zhou J, Mi S (2017) Prevention of cholesterol gallstone disease by schaftoside in lithogenic diet-induced C57BL/6 mouse model. Eur J Pharmacol 815:1-9. https://doi.org/10. 1016/j.ejphar.2017.10.003

Liu X, Cheng J, Zhang G, Ding W, Duan L, Yang J, Kui L, Cheng X, Ruan J, Fan W, Chen J, Long G, Zhao Y, Cai J, Wang W, Ma Y, Dong Y, Yang S, Jiang H (2018) Engineering yeast for the production of breviscapine by genomic analysis and synthetic biology approaches. Nat Commun 9:448. https:// doi.org/10.1038/s41467-018-02883-z

Malla S, Pandey RP, Kim B, Sohng J (2013) Regiospecific modifications of naringenin for astragalin production in Escherichia coli. Biotechnol Bioeng 110:2525-2535. https://doi.org/10.1002/bit.24919

Melo GOD, Muzitano MF, Legora-Machado A, Almeida TA, De Oliveira DB, Kaiser CR, Koatz VL, Costa SS (2005) C-glycosylflavones from the aerial parts of Eleusine indica inhibit LPS-induced mouse lung inflammation. Planta Med 71:362-363. https://doi.org/10.1055/s-2005-864104

Pandey RP, Malla S, Simkhada D, Kim BG, Sohng JK (2013) Production of 3-O-xylosyl quercetin in Escherichia coli. Appl Microbiol Biotechnol 97:1889-1901. https://doi.org/10.1007/s00253-012-4438-9

Pei J, Dong P, Wu T, Zhao L, Fang X, Cao F, Tang F, Yue Y (2016) Metabolic engineering of Escherichia coli for astragalin biosynthesis. J Agric Food Chem 64:7966-7972. https://doi.org/10.1021/acs.jafc.6b03447

Putkaradze N, Teze D, Fredslund F, Welner DH (2021) Natural product C-glycosyltransferases - a scarcely characterised enzymatic activity with biotechnological potential. Nat Prod Rep 38:432-443. https://doi.org/10. 1039/d0np00040j

Ramarathnam N, Osawa T, Namiki M, Kawakishi S (1989) Chemical studies on novel rice hull antioxidants. 2. Identification of isovitexin, a C-glycosyl flavonoid. J Agric Food Chem 37:316-319. https://doi.org/10.1021/jf000 $86 \mathrm{a} 009$

Schmidt S, Rainieri S, Witte S, Matern U, Martens S (2011) Identification of a Saccharomyces cerevisiae glucosidase that hydrolyzes flavonoid glucosides. Appl Environ Microbiol 77:1751-1757. https://doi.org/10.1128/ AEM.01125-10

Shrestha A, Pandey RP, Dhakal D, Parajuli P, Sohng JK (2018) Biosynthesis of flavone C-glucosides in engineered Escherichia coli. Appl Microbiol Biotechnol 102:1251-1267. https://doi.org/10.1007/s00253-017-8694-6

Simkhada D, Lee H, Sohng J (2010) Genetic engineering approach for the production of rhamnosyl and allosyl flavonoids from Escherichia coli. Biotechnol Bioeng 107:154-162. https://doi.org/10.1002/bit.22782

Stevenson PC, Kimmins FM, Grayer RJ, Raveendranath S (1996) Schaftosides from rice phloem as feeding inhibitors and resistance factors to brown planthoppers, Nilaparvata lugens. Entomol Exp Appl 80:246-249. https:// doi.org/10.1111/j.1570-7458.1996.tb00928.x 
Sun Y, Chen Z, Yang J, Mutanda I, Li S, Zhang Q, Zhang Y, Zhang Y, Wang Y (2020) Pathway-specific enzymes from bamboo and crop leaves biosynthesize anti-nociceptive C-glycosylated flavones. Commun Biol 3:110. https://doi.org/10.1038/s42003-020-0834-3

Talhi O, Silva AMS (2012) Advances in C-glycosylflavonoid Research. Curr Org Chem 16:859-896. https://doi.org/10.2174/138527212800194791

Vanegas KG, Larsen AB, Eichenberger M, Fischer D, Mortensen UH, Naesby M (2018) Indirect and direct routes to C-glycosylated flavones in Saccharomyces cerevisiae. Microb Cell Fact 17:107. https://doi.org/10.1186/ s12934-018-0952-5

Wang Z, Gao H, Wang S, Zhang M, Chen K, Zhang Y, Wang H, Han B, Xu L, Song T, Yun C, Qiao X, Ye M (2020) Dissection of the general two-step di-C-glycosylation pathway for the biosynthesis of (iso)schaftosides in higher plants. Proc Natl Acad Sci USA 117:30816-30823. https://doi.org/ 10.1073/pnas.2012745117
Yang Z, Nakabayashi R, Mori T, Takamatsu S, Kitanaka S, Saito K (2016) Metabolome analysis of Oryza sativa (rice) using liquid chromatography-mass spectrometry for characterizing organ specificity of flavonoids with antiinflammatory and anti-oxidant activity. Chem Pharm Bull 64:952-956. https://doi.org/10.1248/cpb.c16-00180

Zhang M, Li F, Li K, Wang Z, Wang Y, He J, Su H, Zhang Z, Chi C, Shi X, Yun C, Zhang Z, Liu Z, Zhang L, Yang D, Ma M, Qiao X, Ye M (2020) Functional characterization and structural basis of an efficient di-C-glycosyltransferase from Glycyrrhiza glabra. J Am Chem Soc 142:3506-3512. https:// doi.org/10.1021/jacs.9b12211

\section{Publisher's Note}

Springer Nature remains neutral with regard to jurisdictional claims in published maps and institutional affiliations.

\section{Submit your manuscript to a SpringerOpen ${ }^{\circ}$ journal and benefit from:}

- Convenient online submission

- Rigorous peer review

- Open access: articles freely available online

- High visibility within the field

- Retaining the copyright to your article

Submit your next manuscript at $\boldsymbol{\nabla}$ springeropen.com 